\title{
Thermodynamics of Nucleation of Silicon Carbide Nanocrystals during Carbonization of Porous Silicon
}

\author{
Yu.S. Nagornov* \\ Institute of Microstructure Technology, Karlsruhe Institute of Technology, Hermann-von-Helmholtz-Platz 1, 76344 \\ Eggenstein-Leopoldshafen, Germany
}

(Received 14.04.2016; published online 03 October 2016)

\begin{abstract}
The formation of $\mathrm{SiC}$ nanocrystals of the cubic modification in the process of high-temperature carbonization of porous silicon has been analyzed. It has been shown that the surface energy of silicon nanoparticles and quantum filaments is released in the process of annealing and carbonization. The Monte Carlo simulation has shown that the released energy makes it possible to overcome the nucleation barrier and to form $\mathrm{SiC}$ nanocrystals.
\end{abstract}

Keywords: Thermodynamics of nucleation, $\mathrm{SiC}$ nanocrystals, High-temperature carbonization, Porous silicon, Monte Carlo simulation.

DOI: 10.21272/jnep.8(3).03001

PACS numbers: 82.60.Nh, 81.07.Bc, 64.70.Nd

\section{INTRODUCTION}

The investigation of the thermodynamic characteristics of a phase transition in multicomponent media or media with a developed open surface is an important fundamental problem of modern physics [1-3]. The authors of recent works on heterogeneous melting [1,3] considered a model of dynamics of melting of thin metal films on silicon and silicon oxide, where the surface was considered as a thin layer of a supercooled melt or an adsorption layer reducing the surface energy of a crystalline phase. In classical thermodynamics, the transition temperature $T_{0}$ from a solid to a liquid state is the temperature at which the Gibbs potential of the liquid phase is equal to that of the solid phase. The process of melting on the surface occurs at temperatures much lower than the temperature of the phase transition in a bulk material; at these temperatures, nanocrystals and quantum filaments are melted [1-4]. As a result, a thermodynamically stable state-liquid supercooled layerappears on the surface; for this layer, from the equality of the Gibbs potentials for the solid and liquid states with the inclusion of the surface energy and under the assumption of the equality of the entropies of the phase transition on the surface and in the bulk [5-6].

The consideration of the thermodynamics of melting of surface films made it possible to describe experimental studies of the dynamics of the rolling and melting of metal films on inert surfaces, as well as the melting of metal nanoparticles in microchannel structures, and to refine the mechanism of heteroepitaxial growth and the growth of carbon nanotubes [3, 7-9]. The application of this thermodynamic approach to porous media with a developed surface is of fundamental and applied interest. One of the most well studied porous materials is porous silicon (por-Si) for which there are many experimental data on photoluminescence and the structure and the mechanism of the electrochemical etching and formation of silicon nanocrystals is well studied [10-13].

The formation of ( $\mathrm{SiC}$ nanocrystal-Si nanocrystal) heterostructures at the carbonization of por-Si was pointed out in $[13,14]$, but the mechanism of this phenomenon was not revealed. The aim of this work is to apply the proposed thermodynamic approach to explain the behavior of silicon nanoparticles in the process of high-temperature carbonization.

\section{THERMODYNAMICS OF NUCLEATION OF SILICON CARBIDE NANOCRYSTALS}

The surface energy of silicon nanoparticles in the process of high-temperature carbonization can be spent on the overcoming of the nucleation barrier at the formation of silicon carbide nanocrystals [6]. According to the theory of nucleation [15], where a phase transition under nearly equilibrium conditions is considered, a change in the Gibbs free energy $\Delta G$ of a nucleus of a new phase at a finite temperature $T$ is

$$
\Delta G=V\left(\Delta g+\varepsilon_{V}\right)+\sum \sigma S,
$$

where $V$ is the volume of the nucleus; $S$ and $\sigma$ are the area of the surface and the surface energy of the corresponding face, respectively; $\Delta g$ is the specific change in the Gibbs energy per unit volume disregarding the surface energy determined by the degree of supercooling of the system; and $\varepsilon V$ is the specific elastic energy. The surface energy for a spherical nucleus is given by the product $\sigma S$, where $\sigma$ is the surface energy averaged over all directions. It is known [15] that the function $\Delta G$ has a maximum at the critical radius of the nucleus $r_{c}$.

Using the known enthalpies of formation of silicon and silicon carbide cubic crystals $\Delta H(\mathrm{c}-\mathrm{Si})=$ $-45.5 \mathrm{~kJ} / \mathrm{mol}$ and $\Delta H(3 \mathrm{C}-\mathrm{SiC})=-62.7 \mathrm{~kJ} / \mathrm{mol}[16]$ and taking into account that the transition from the crystalline state of silicon to the cubic crystalline state of silicon carbide occurs at a finite temperature, one can find the value of $\Delta g$. The temperature determines the rate of the phase transition, whereas the energy of transition is determined only by the difference between the enthalpies.

\footnotetext{
*Nagornov.Yuri@gmail.com
} 

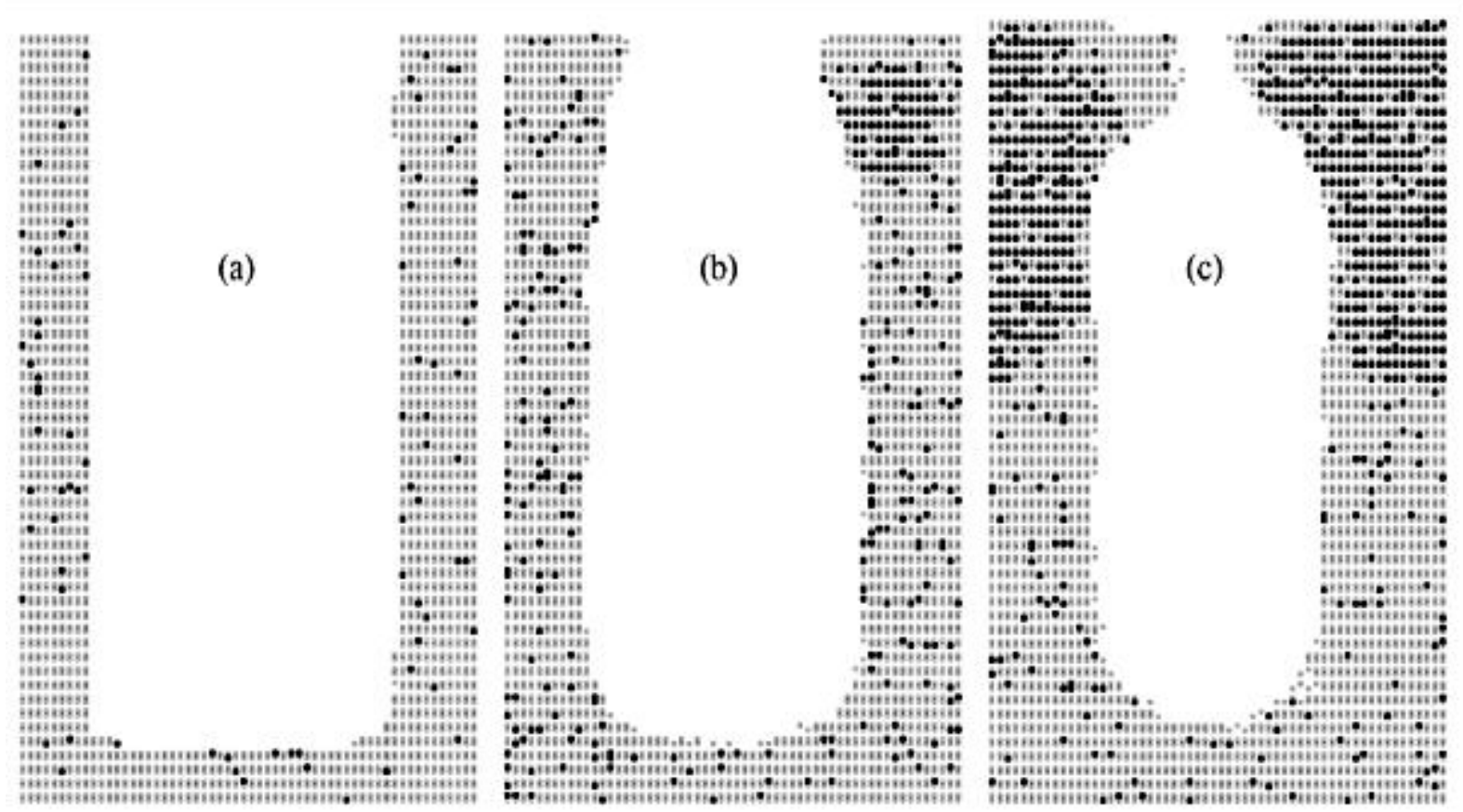

Fig. 1 - Dynamics of the carbonization of porous silicon at a temperature of $1273 \mathrm{~K}$ at times $t=$ (a) 1 , (b) 7 , and (c) $14 \mathrm{~s}$; the depth and thickness of the porous layer are 15 and $4.5 \mathrm{~nm}$, respectively. Silicon and carbon atoms are shown by light and black points, respectively $[6,16]$

In the case under consideration, carbonization occurs at temperatures when silicon filaments in por-Si are in a liquid state [17]. Consequently, the elastic energy $\varepsilon V$ can be disregarded in Eq. (1). Thus, to calculate the critical radius, it is necessary to know the average surface energy of the 3C-SiC crystal. For definiteness, a surface energy of $2.18 \mathrm{aJ} / \mathrm{nm}^{2}$ obtained experimentally in $[6,18]$ is used.

The critical radius and energy of nucleation were determined in [6] for various surface energies and temperatures of carbonization. The critical radius of a nucleus and the free energy of the barrier with an increase in the temperature from 1073 to $1573 \mathrm{~K}$ vary from 3.1 to $4.1 \mathrm{~nm}$ and from 550 to $976 \mathrm{eV}$, respectively. It is clear that, for the creation of a silicon carbide nucleus of the cubic modification, the system should overcome the energy barrier $\Delta G_{c}$ and a carbon atomic flux to the surface of the nucleus should be created for the growth of the nucleus.

Figures 1 and 2 show the results of the Monte Carlo simulation of the nucleation dynamics of silicon carbide in the process of carbonization of por-Si $[6,16]$ with the algorithm described in [19-21]. The numerical simulation reveals several interesting features of the formation of silicon carbide nanocrystals. First, nanocrystals are formed only in silicon quantum filaments and are not formed in a single crystal substrate in agreement with the experimental data $[6,17,22]$. Second, the stoichiometric coefficient $k_{S i C}$ depends on the carbonization temperature: $k_{S i C}=0.8$ at $1273 \mathrm{~K}$ and $k_{S i C}=0.6-0.7$ at $1173 \mathrm{~K}[16,22]$. Furthermore, at a significant reduction of the temperature (down to $1073 \mathrm{~K}$ and below), silicon carbide nanocrystals are not formed. Third, the formation of silicon carbide nanocrystals occurs in times an order of magnitude smaller than the time of the collapse of pores and quantum filaments in the process of annealing [23]. The calculations show that nanocrystals are formed in times of 5-15 s.

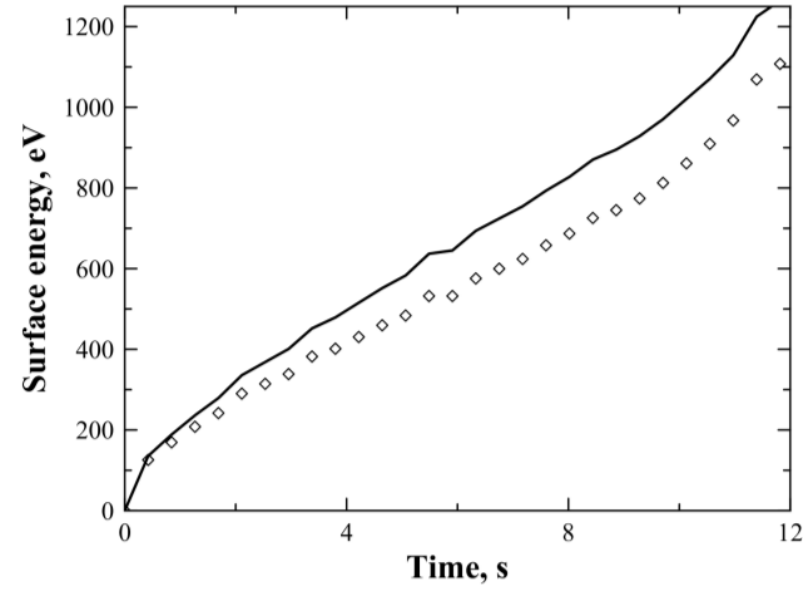

Fig. 2 - Dynamics of the surface energy release of one pore with a width of $4.5 \mathrm{~nm}$ and a depth of $15 \mathrm{~nm}$ in the process of carbonization at a temperature of $1273 \mathrm{~K}$. The points show the calculation disregarding a change in the structure of the surface and the line is the calculation including a change in the structure of the surface over the (111) plane

In the numerical experiment, the rate of deposition was one or two atomic layers per second, which corresponds to the epitaxial growth of the crystal. In the case of shallow pores (up to $45 \mathrm{~nm}$ ), a nanocrystal in the quantum filament is formed near the tops of pores and quantum filaments [6, 16] (Fig. 1). The dynamics of formation of silicon carbide inside a quantum filament consists of several steps [22]: the deposition of carbon atoms, surface diffusion along the walls of pores and quantum filaments, bulk diffusion into quantum filaments (Fig. 1a), the formation of a silicon carbide cluster 
at the center of the quantum filament (Fig. 1b) and its further growth, the closure of pores in the process of annealing, and the melting of quantum filaments (Fig. 1c).

In the numerical experiment, in order to estimate the role of the surface energy, the surface energy and the difference between its initial value and the current value, i.e., the surface energy release were calculated (Fig. 2). The calculations $[6,16]$ showed that the surface diffusion in the initial time period (to $4 \mathrm{~s}$ ) is larger than the flux to the bulk of porous silicon by a factor of 80 100. Then (from 4 th to 12 th second), several processes proceed simultaneously: tops of pores overlap because of the melting of silicon quantum filaments and the total area decreases to half of the initial area [16] for two reasons: first, owing to a melting-induced decrease in the area of silicon quantum filaments and, second, because of the deposition of carbon atoms on the tops of quantum filaments, which leads to an increase in the volume of silicon quantum filaments, as well as to overlapping and filling of pores. The initial period of carbonization includes the formation of silicon carbide nanocrystals (see Fig. 1) and overcoming of the nucleation barrier owing to a change in the surface energy [6].

A change in the surface energy of quantum filaments is numerically estimated for two extreme cases (Fig. 2). In the first case, it was assumed that the structure of the surface does not change and only the area of the surface varies. In the second case, it was assumed that the structure of the surface changes sharply toward the $\{111\}$ direction, so that its fraction at the time $t=12 \mathrm{~s}$ is $50 \%$; for definiteness, it was accepted that changes are linear. The surface energy in the process of carbonization is correspondingly between these two extreme cases.

According to calculations in [6], the nucleation barrier energy $\Delta G_{c}=682 \mathrm{eV}$ at a temperature of $1273 \mathrm{~K}$ is released in $7-9 \mathrm{~s}$ (Fig. 2). It is seen in Fig. 3 that a silicon carbide nanocrystal is formed at this time, which is implicitly confirmed by the expenditure of the surface energy for overcoming the nucleation barrier [6, 23]. Taking into account diffusion processes in the system including single crystal silicon and carbon atoms, one can explain why silicon carbide nanocrystals are not formed at low temperatures. In the process of hightemperature carbonization of porous silicon, an additional energy appears owing to a decrease in the surface

\section{REFERENCES}

1. U. Tartaglino, T. Zykova-Timan, F. Ercolessi, E. Tosatti, Phys. Rep. 411, 291 (2005).

2. P. Muller, R. Kern, Surf. Sci. 529, 59 (2003).

3. D.G. Gromov, S.A. Gavrilov, Phys. Solid State 51 (10), 2135 (2009).

4. C.C. Yang, G. Li, Q. Jiang, J. Phys.: Condens. Matter 15, 4961 (2003).

5. Yu.S. Nagornov, Tech. Phys. Lett. 41 (6), 532 (2015).

6. Yu.S. Nagornov, Tech. Phys. 60 (5), 700 (2015).

7. D.G. Gromov, S.A. Gavrilov, E.N. Redichev, R.M. Ammosov, Phys. Solid State 49 (1), 178 (2007).

8. A.A. Buzdugan, S.A. Gavrilov, D.G. Gromov, E.N. Redichev, I.S. Chulkov, Izv. Vyssh. Uchebn.Zaved., Elektron. 2, 21 (2007).

9. M. Wautelet, J.P. Dauchot, M. Hecq, Nanotechnology 11, 6 energy of pores. As a result, the thermodynamic system of carbon and silicon atoms acquires energy for the formation of a nucleus. The surface energy at a temperature of $1273 \mathrm{~K}$ is released in $7-8 \mathrm{~s}$, whereas the same energy at lower temperatures is released several orders of magnitude more slowly [22, 24]. Since the diffusion coefficient of silicon at temperatures above $1073 \mathrm{~K}$ is larger than that of silicon [24], carbon atoms at such temperatures penetrate into the bulk of a silicon quantum filament and use the surface energy release to form a nucleus. On the contrary, carbon atoms at temperatures below $1073 \mathrm{~K}$ do not have time to create supersaturation inside the silicon quantum filament and do not use the surface energy of pores. For this reason, the stoichiometric coefficient $k_{S i C}$ depending on the carbonization temperature $[16,22]$ decreases with a decrease in this temperature.

Another reason is that the dynamics of transformation of the surface of pores, as well as the rate of formation of nuclei, decreases exponentially by several orders of magnitude with a decrease in the temperature, whereas the scattering of the energy depends only on the temperature gradient and varies linearly. As a result, a fraction of the surface energy release is scattered to the environment and, the lower the carbonization temperature and annealing, the larger the scattered fraction of the energy.

\section{CONCLUSION}

Thus the formation of silicon carbide nanocrystals in the process of high- temperature carbonization of por-Si has been analyzed within the model of heterogeneous surface melting. The distribution of particles over transverse dimensions for initial porous silicon after hightemperature treatment has been obtained. It has been shown that the surface melting of nanoparticles smaller than $70 \mathrm{~nm}$ occurs at a temperature of $1573 \mathrm{~K}$; silicon carbide nanocrystals of the cubic modification are formed in these nanoparticles. The Monte Carlo simulation has shown that silicon carbide particles have an imperfect structure and consist of several nanocrystals. At the time of the formation of the 3C-SiC nanocrystals, the surface energy released in the process of carbonization corresponds the nucleation barrier for the thermodynamic system consisting of silicon and silicon carbide of the cubic modification.
(2000).

10. E.A. Agafonova, M.N. Martyshov, P.A. Forsh, V.Yu. Timoshenko, P.K. Kashkarov, Semiconductors 44 (3), 350 (2010)

11. D.M. Zhigunov, N.V. Shvydun, A.V. Emel'yanov, V.Yu. Timoshenko, P.K. Kashkarov, V.N. Seminogov, Semiconductors 46 (3), 354 (2012).

12. B.M. Kostishko, A.V. Zolotov, Yu.S. Nagornov, Conference "Silicon, School-2001" (Chernogolovka: Moscow oblast, Russia: 2001).

13. B.M. Kostishko, Sh.R. Atazhanov, I.P. Puzov, S.Ya. Solomatin, Yu.S. Nagornov, Tech. Phys. Lett. 26 (3), 199 (2000).

14. B.M. Kostishko, Yu.S. Nagornov, S.Ya. Salomatin, Sh.R. Atazhanov, Tech. Phys. Lett. 30 (2), 88 (2004). 
15. A.R. West, Solid State Chemistry and Its Applications (Wiley: New York: 1987; Mir: Moscow: 1988).

16. A.V. Zolotov, Candidate's Dissertation in Mathematical Physics (Ulyanovsk State University, Ulyanovsk, 2007).

17. Yu.S. Nagornov, B.M. Kostishko,

S.N. Mikov, Sh.R. Atazhanov, A.V. Zolotov, Tech. Phys. 52 (8), 1093 (2007).

18. B.N. Oshcherin, phys. status solidi a 34, K181 (1976).

19. S. Kersulis, V. Mitin, Semicond. Sci. Technol. 10, 653 (1995).
20. A.V. Zverev,

I.G. Neizvestnyi,

N.L. Shvarts,

Z.Sh. Yanovitskaya, Semiconductors 35 (9), 1022 (2001).

21. N. Ott, M. Nerding, G. Muller, phys. status solidi a 197, 93 (2003).

22. Yu.S. Nagornov, B.F. Mel'nikov, A.V. Zolotov, Vektor Nauki Tolyatti Gos. Univ. 4 (22), 89 (2012).

23. B.M. Kostishko, A.V. Zolotov, Yu.S. Nagornov, Semiconductors 43 (3), 355 (2009)

24. Handbook of Physical Quantities (Ed. by I.S. Grigoriev, E.Z. Meilikhov) (Energoatomizdat, Moscow: 1991; CRC Press: Boca Raton, Florida, United States: 1996). 